\title{
Predictive Role of De Ritis Ratio in Biochemical Recurrence After Radical Prostatectomy
}

\section{Radikal Prostatektomi Sonrası Biyokimyasal Rekürrens Tahmininde De Ritis Oranının Rolü}

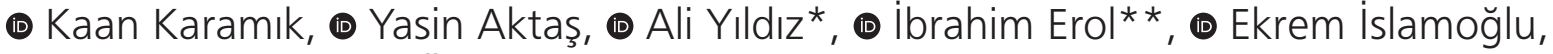 \\ ๑ Mutlu Ateş, ๑ Taha Ölçücü, ๑ Kayhan Yılmaz, ๑ Murat Savaş \\ University of Health Sciences Antalya Training and Research Hospital, Clinic of Urology, Antalya, Turkey \\ *Varto State Hospital, Clinic of Urology, Muş, Turkey \\ ${ }^{*}$ Kastamonu State Hospital, Clinic of Urology, Kastamonu, Turkey
}

\section{Abstract}

Aim: To analyze the role of De Ritis (aspartate aminotransferase/ alanine aminotransferase) ratio in predicting biochemical recurrence $(B C R)$ after radical prostatectomy (RP).

Methods: We retrospectively evaluated 425 patients with localised prostate cancer who underwent RP from 2009 to 2018. Patients with neo-adjuvant treatment, elevated liver enzymes, postoperative early hormone therapy, incomplete clinicopathological data and a follow-up of less than 6 months were excluded from the study. Demographic, clinical, pathological and follow-up data of the patients were recorded. Patients with and without BCR were compared. Sensitivity and specificity of De Ritis ratio in predicting BCR were calculated.

Results: According to the maximum value of the Youden index, optimal threshold of De Ritis ratio for BCR was 1.1. Sensitivity, specificity, positive predictive value and negative predictive value were $69.7 \%, 61.1 \%, 37.6 \%$ and $85.7 \%$, respectively. Multivariate analysis showed that the De Ritis ratio ( $H R=1.968, p=0.014)$ was a significant predictor factor for BCR. BCR-free survival rate was significantly lower in patients with higher De Ritis ratio.

Conclusion: Our study suggests that elevated De Ritis ratio and detailed pathological findings could be an independent predictive factor for BCR after RP.

Keywords: De Ritis ratio, prostate cancer, biochemical recurrence, pathological findings, biochemical recurrence-free survival
Amaç: Radikal prostatektomi (RP) sonrası biyokimyasal rekürrensi (BR) tahmin etmede De Ritis (aspartat aminotransaminaz/alanin aminotransaminaz) oranının rolünü analiz etmektir.

Yöntemler: 2009-2018 yılları arasında lokalize prostat kanseri nedeni ile RP yapılan 425 hasta retrospektif olarak incelendi. Neoadjuvan tedavi alan, karaciğer enzim yüksekliği olan, ameliyat sonrası erken dönem hormon tedavisi alan, klinikopatolojik verileri eksik olan ve takip süresi 6 aydan kısa olan hastalar çalışma dışı bırakıldı. Hastaların demografik, klinik, patolojik ve takip verileri kaydedildi. BR olan ve olmayan hastalar karşılaştırıldı. BR tahmin etmede De Ritis oranının sensitivite ve spesifitesi hesaplandı.

Bulgular: Maksimum Youden indeks değerine göre, BR için optimal De Ritis oranı eşiği 1,1 olarak hesaplandı. Bu değere göre sensitivite $\% 69,7$, spesifite $\% 61,1$, pozitif prediktif değer \%37,6, negative prediktif değer \%85,7 olarak tespit edildi. BR'yi öngören faktörler için çok değişkenli analizde De Ritis oranı $(H R=1,968, p=0,014)$ anlamlı değişken olarak tespit edildi. BR'siz sağkalım yüksek De Ritis oranına sahip hastalarda daha kısa olarak belirlendi.

Sonuç: Çalışmamız, artmış De Ritis oranının ve ayrıntılı patolojik bulguların RP sonrası BR için bağımsız bir öngörücü faktör olabileceğini düşündürmektedir.

Anahtar Sözcükler: De Ritis oranı, prostat kanseri, biyokimyasal rekürrens, patolojik bulgular, biyokimyasal rekürrenssiz sağkalım
Address for Correspondence/Yazışma Adresi: Kaan Karamık, University of Health Sciences Antalya Training and Research Hospital, Clinic of Urology, Antalya, Turkey

Phone: +90 5544876649 E-mail: kaankaramik@gmail.com ORCID: orcid.org/0000-0001-8288-5313

Received/Geliş Tarihi: 17 September 2019 Accepted/Kabul Tarihi: 06 December 2019

This study was presented at the $9^{\text {th }}$ Eurasian Urooncology Congress, 16-20 October 2019, Athens, Greece.
${ }^{0}$ Copyright 2020 by The Medical Bulletin of istanbul Haseki Training and Research Hospital The Medical Bulletin of Haseki published by Galenos Yayınevi. •Telif Hakkı 2020 istanbul Haseki Eğitim ve Araştırma Hastanesi Haseki Tıp Bülteni, Galenos Yayınevi tarafından yayınlanmıştır. 


\section{Introduction}

Prostate cancer (PCa) is the most frequently diagnosed solid cancer and the second leading cause of death among men (1). Widespread use of prostate specific antigen (PSA) screening has resulted in an increased number of patients diagnosed with localised PCa. At a median follow-up of ten years, the oncologic outcomes of radical prostatectomy (RP) and external beam radiotherapy have been reported to be equal (2). Nevertheless, RP has been shown to provide tumor control, accurate staging and elimination of possible PSA sources. Patients are monitored for failure with repeated PSA testing after RP. However, biochemical recurrence (BCR) has been observed in approximately 35\% patients after surgery (3). In a study including only low-risk PCa patients, the rate of BCR was found to be $25 \%$ (4). Patients who experience $B C R$, which can require secondary therapy, have poorer oncological outcomes. In this sense, identifying patients at high risk for BCR following RP is essential for treatment and follow-up. Several clinical and pathological findings, such as preoperative PSA levels, pathological stage, high Gleason score (GS) and positive surgical margin (PSM), are well-known predictors of BCR (5-7). Apart from these factors, in recent years some authors suggested to illustrate some other markers, systemic inflammation biomarkers, such as neutrophil-tolymphocyte ratio and detailed pathological findings such as perineural invasion (PNI) and lymphovascular invasion (LVI) (8-10). Thus, risk assessment is of immense value for patient counseling prior to treatment decision.

Aminotransaminases, including aspartate aminotransaminase (AST) and alanine aminotransaminase (ALT), are the most commonly used liver enzymes. They are produced by both malignant and non-malignant cells. The AST-to-ALT ratio, also termed the De Ritis ratio, was initially described by De Ritis in 1957 (11). Although it has been used as an indicator for viral hepatitis and several chronic liver diseases, recently De Ritis ratio has been identified as an independent predictor of patient survival in certain types of malignancies (12-15). In this study, our main aim was to evaluate whether the De Ritis ratio and previously known clinicopathological parameters were significant predictors for BCR after RP.

\section{Methods}

\section{Study Design}

Data belonging to 425 patients who underwent RP for PCa, without neo-adjuvant treatment, at our institution from April 2009 to April 2018 were retrospectively reviewed. We excluded 123 patients from analysis, because 23 patients had previously diagnosed or preoperatively detected liver diseases (e.g., non-alcoholic fatty liver diseases, chronic hepatitis, liver cirrhosis), four patients received postoperative early hormone therapy, 62 patients had a follow-up time shorter than 6 months, 30 patients had incomplete clinicopathological data and four patients had persistent PSA after RP. While open retropubic RP was performed using the Walsh technique (16) by three different surgeons, robot-assisted laparoscopic prostatectomy was performed with da Vinci robotic surgical system (Intuitive Surgical, Sunnyvale, CA, USA) by two of the three surgeons. Patients with a lymph node metastasis risk of above $5 \%$ according to the Briganti nomogram (17) had extended lymph node dissection performed.

The patients' age and clinical history, preoperative PSA value, digital rectal examination (DRE) findings, biopsy GS, clinical T stage, postoperative GS, pathological T stage, tumor volume percent, surgical margin status, presence of PNI and LVI, extraprostatic extension (EPE), seminal vesicle invasion (SVI), presence of lymph node metastasis, the AST and ALT levels, and the De Ritis ratio were pooled from the database and analyzed. Risk stratification was performed according to D'Amico risk classification (18).

\section{Evaluation of De Ritis Ratio}

AST and ALT levels were routinely measured by spectrophotometric method (Beckman Coulter ${ }^{\circledR}$ AU5800) 3-7 days before surgery. A level of $\geq 50 \mathrm{IU} / \mathrm{L}$ was defined as upper reference level for AST and ALT.

\section{Postoperative Follow-up}

The patients were followed up postoperatively with PSA every 3 months for the first year, every 6 months for the second year and then annually thereafter. BCR was defined by two consecutive PSA levels $\geq 0.2 \mathrm{ng} / \mathrm{mL}$. BCRfree survival was calculated from the time of RP to $B C R$.

\section{Statistical Analysis}

Statistical analysis was made using the IBM SPSS Statistics for Windows, version 23.0 (IBM Corp., Armonk, NY). Fisher's exact test and Pearson chi-square test were performed for categorical variables. The normality assumptions were controlled by the Shapiro-Wilk test. Differences between two groups were evaluated with Student's t-test for normally distributed data or the Mann-Whitney $U$ test for non-normally distributed data. The Kruskal-Wallis test was used for comparison of nonparametric variables between groups and the BonferroniDunn test was used as a post-hoc test for significant cases. The receiver operating characteristic (ROC) curve analysis was applied to evaluate predictive performance of De Ritis ratio in determining $B C R$ and non-BCR patients and area under the curve (AUC), sensitivity and specificity were calculated and reported with 95\% confidence intervals. Youden's index was calculated to determine the optimal cut-off values. The Kaplan-Meier method and log-rank 
tests were used to determine survival differences for nominal variables. A multivariate Cox proportional hazards regression model was used to identify independent prognostic factors for BCR. Hazard ratio (HR), with corresponding $95 \%$ confidence intervals $(95 \% \mathrm{Cls})$, was reported. All prognostic factors that were significant on univariate analysis were analyzed in the multivariate model. Data are expressed as $n(\%)$, mean \pm standard deviation or median (min-max), as appropriate. A p value of less than 0.05 was considered statistically significant.

\section{Results}

Baseline demographic and clinical characteristics are summarized in Table 1. The mean age at the time of surgery was $63.9 \pm 6.3$ years. The median PSA value was $8.18 \mathrm{ng} / \mathrm{mL}$ (3.06-107.32) during a median follow-up of 16 months (6-108). BCR was determined in 76 patients $(25.2 \%)$. PSM was detected in 93 patients $(30.8 \%)$. The median AST and ALT values were 21 (12-44) and 18 (648) IU/L, respectively. The median De Ritis ratio was 1.08 (0.6-3). De Ritis ratio cutoff value was set at 1.1, and the patients were categorized into two groups $(\leq 1.1$ and $>1.1)$. A ROC analysis was performed to predict $B C R$, and the AUC value was 0.605 (0.547-0.660) (Figure 1). Using the Youden's index for cut-off point, sensitivity, specificity, positive predictive value and negative predictive value were $69.7 \%, 61.1 \%, 37.6 \%$ and $85.7 \%$, respectively.

The relationship of BCR with preoperative clinicopathological parameters, De Ritis ratio and postoperative pathological findings after RP is shown in Table 2. PSA values, DRE, biopsy GS, D'Amico risk classification, clinical T stage, De Ritis ratio, postoperative GS, pathological T stage, tumor volume percent, PSM, PNI, $\mathrm{LVI}, \mathrm{EPE}, \mathrm{SVI}$, lymph node involvement (LNI), multifocal PSM and upgrading were found to be statistically significant, whereas age, prostate weight, ALT and AST were not found to be related with $B C R$.

We also examined the impact of De Ritis ratio and clinicopathological findings on the prediction of BCR. On univariate Cox proportional hazards model, initial PSA $(H R=1.034, p<0.001)$, intermediate risk $(H R=4.391$, $p<0.001)$ and high risk $(H R=10.679, p<0.001)$ patients according to D'Amico, positive DRE ( $H R=5.752$, $p<0.001)$, De Ritis ratio $(H R=2.853, p<0.001)$, clinical $T$ stage $\geq 2(H R<.4 .743, p<0.001)(H R=27.877, p<0.001)$, postoperative $\mathrm{GS} \geq 7 \quad(\mathrm{HR}=5.018, \quad \mathrm{p}<0.001)(\mathrm{HR}=9.972$, $p<0.001)$, pathological T stage $\geq 3(H R=6.637, p<0.001)$, PNI (HR=6.38, $p=0.002), \quad L V I \quad(H R=5.052, p<0.001)$, EPE $(H R=6.601, p<0.001), \quad S V I \quad(H R=6.466, p<0.001)$, LNI ( $H R=7.357, p<0.001)$, multifocal PSM ( $H R=5.332$, $p<0.001)$, PSM $(H R=4.046, p<0.001)$, and upgrading $(H R=2.662, \quad p<0.001)$ were significant predictors for

\begin{tabular}{|c|c|}
\hline Variables & $\mathrm{n}=302$ \\
\hline Age (years) (mean \pm SD) & $63.9 \pm 6.3$ \\
\hline PSA (ng/mL) (median) (min-max) & $8.18(3.06-107.32)$ \\
\hline \multicolumn{2}{|l|}{ PSA distributions, n (\%) } \\
\hline$<10$ & $190(62.9)$ \\
\hline $10-20$ & $73(24.2)$ \\
\hline$>20$ & 39 (12.9) \\
\hline \multicolumn{2}{|l|}{ Digital rectal examination, $\mathrm{n}(\%)$} \\
\hline Positive & $138(45.7)$ \\
\hline Negative & $164(54.3)$ \\
\hline \multicolumn{2}{|l|}{ Biopsy GS, n (\%) } \\
\hline$\leq 6$ & $207(68.5)$ \\
\hline 7 & $76(25.2)$ \\
\hline$\geq 8$ & $19(6.3)$ \\
\hline \multicolumn{2}{|l|}{ D'Amico risk classification, $\mathrm{n}(\%)$} \\
\hline Low & $150(49.7)$ \\
\hline Intermediate & $99(32.8)$ \\
\hline High & $53(17.5)$ \\
\hline \multicolumn{2}{|l|}{ Clinical stage, n (\%) } \\
\hline T1a & $0(0)$ \\
\hline $\mathrm{T} 1 \mathrm{~b}$ & $1(0.3)$ \\
\hline T1c & $164(54.3)$ \\
\hline $\mathrm{T} 2 \mathrm{a}$ & $75(24.8)$ \\
\hline $\mathrm{T} 2 \mathrm{~b}$ & $25(8.3)$ \\
\hline $\mathrm{T} 2 \mathrm{C}$ & $29(9.6)$ \\
\hline ТЗа & $8(2.6)$ \\
\hline T3b & $0(0)$ \\
\hline ALT (IU/L) (median) (min-max) & $18(6-48)$ \\
\hline AST (IU/L) (median) (min-max) & $21(12-44)$ \\
\hline De Ritis ratio & $1.08(0.6-3)$ \\
\hline \multicolumn{2}{|l|}{ Surgical techique, n (\%) } \\
\hline Open retropubic & $109(36.1)$ \\
\hline Robotic & $193(63.9)$ \\
\hline Prostate Weight (g) (median) (min-max) & $51(20-180)$ \\
\hline \multicolumn{2}{|l|}{ Postoperative GS, n (\%) } \\
\hline$\leq 6$ & $125(41.4)$ \\
\hline 7 & $139(46)$ \\
\hline$\geq 8$ & $38(12.6)$ \\
\hline
\end{tabular}




\begin{tabular}{|c|c|}
\hline Variables & $n=302$ \\
\hline \multicolumn{2}{|l|}{ Pathological Stage, n (\%) } \\
\hline $\mathrm{T} 2 \mathrm{a}$ & $55(18.2)$ \\
\hline $\mathrm{T} 2 \mathrm{~b}$ & $12(4)$ \\
\hline $\mathrm{T} 2 \mathrm{c}$ & $127(42.1)$ \\
\hline ТЗа & $56(18.5)$ \\
\hline T3b & $52(17.2)$ \\
\hline $\begin{array}{l}\text { Tumor Volume Percent } n \text { (\%) (median) } \\
\text { (minimum-maximum) }\end{array}$ & $10(1-95)$ \\
\hline \multicolumn{2}{|l|}{ Surgical margin status, $\mathrm{n}(\%)$} \\
\hline Positive & $93(30.8)$ \\
\hline Negative & $209(69.2)$ \\
\hline \multicolumn{2}{|l|}{ Perineural invasion, n (\%) } \\
\hline Absent & $64(21.2)$ \\
\hline Present & $238(78.8)$ \\
\hline \multicolumn{2}{|l|}{ Lymphovascular invasion, n (\%) } \\
\hline Absent & $243(80.5)$ \\
\hline Present & $59(19.5)$ \\
\hline \multicolumn{2}{|l|}{ Extraprostatic extension, n (\%) } \\
\hline Absent & $206(68.2)$ \\
\hline Present & $96(31.8)$ \\
\hline \multicolumn{2}{|l|}{ Seminal vesicle invasion, $\mathrm{n}(\%)$} \\
\hline Absent & $251(83.1)$ \\
\hline Present & $51(16.9)$ \\
\hline \multicolumn{2}{|l|}{ Lymph node involment, $\mathrm{n}(\%)$} \\
\hline negative & $287(95)$ \\
\hline positive & $15(5)$ \\
\hline \multicolumn{2}{|l|}{ Location of PSM, n (\%) } \\
\hline Apical & $50(16.6)$ \\
\hline Posterior & $10(3.3)$ \\
\hline Posterolateral & $16(5.3)$ \\
\hline Lateral & $9(3)$ \\
\hline Anterior & $2(0.7)$ \\
\hline Bladder neck & $6(2)$ \\
\hline Negative SM & $209(69.2)$ \\
\hline \multicolumn{2}{|l|}{ Multifocal PSM, n (\%) } \\
\hline No & $272(90.1)$ \\
\hline Yes & $30(9.9)$ \\
\hline \multicolumn{2}{|l|}{ Biochemical recurrence, $\mathrm{n}(\%)$} \\
\hline No & $226(74.8)$ \\
\hline Yes & $76(25.2)$ \\
\hline
\end{tabular}

\begin{tabular}{|l|l|}
\hline Table 1. Continued & $\mathbf{n}=\mathbf{3 0 2}$ \\
\hline Variables & $122(40.4)$ \\
\hline Equilavence between biopsy GS and prostatectomy GS, $\mathbf{n}(\%)$ \\
\hline Upgrading & $13(4.3)$ \\
\hline Downgrading & $167(55.3)$ \\
\hline Same GS & score, ALT: Alanine \\
\hline $\begin{array}{l}\text { PSA: Prostate specific antigen, GS: Gleason } \\
\text { aminotransaminase, AST: Aspartate aminotransaminase, PSM: Positive surgical } \\
\text { margin, SD: Standard deviation, n: Number }\end{array}$ \\
\hline
\end{tabular}

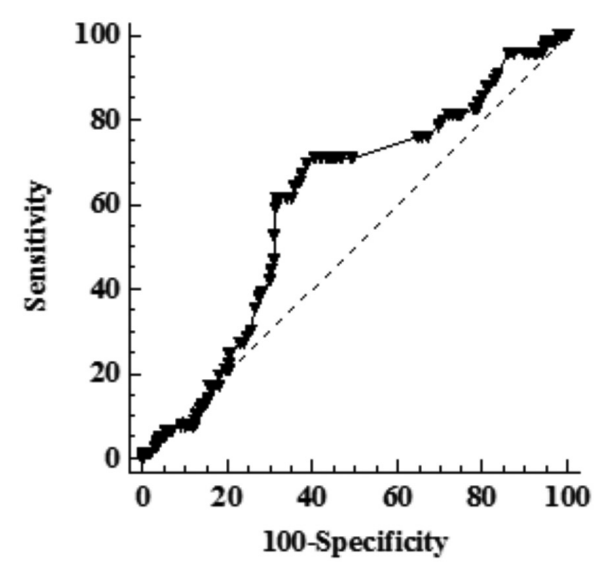

Cutoff $>1.1$, Sensitivity. $69.74(58.1-79.8)$, Specificity. $61.06(54.4-67.5)$, AUC:0.605(0.547$0.660), \mathrm{p}=0.004$

Figure 1. $A$ ROC analysis was performed to predict $B C R$ ROC: Receiving operating characteristic, BCR: Biochemical recurrence, AUC: Area under curve

$B C R$. Multivariate analysis showed that intermediate $(H R=2.228, p=0.037)$ and high $(H R=3.316, p=0.004)$ D'Amico classification, De Ritis ratio $(H R=1.968, p=0.014)$, LVI (HR=1.734, $p=0.045)$, EPE $(H R=1.970, p=0.027), S V I$ $(H R=1.738, p=0.048), P S M(H R=1.986, p=0.008)$ and upgrading $(H R=1.670, \quad p=0.045)$ remained predictor factors for BCR (Table 3).

$B C R$-free survival curves according to eight predictive factors for BCR are shown in Figure 2. Kaplan-Meier estimates of BCR-free survival stratified according to high De Ritis ratio and low De Ritis ratio. $(p<0.001)$ (Figure $2 A)$ $B C R$-free survival rate was significantly lower in patients with PSM than in those having negative surgical margin $(p<0.001)$ (Figure 2B). Furthermore, patients with higher PSA values, postoperative GS $\geq 8$, LVI, PNI, and multifocal PSM were associated with lower BCR-free survival rates.

\section{Discussion}

Our results demonstrated that De Ritis ratio was an independent predictive factor for BCR after RP. Moreover, $B C R$-free survival rates were significantly lower in patients 


\begin{tabular}{|c|c|c|c|}
\hline \multirow[t]{2}{*}{ Variables } & \multicolumn{2}{|l|}{ BCR } & \multirow[b]{2}{*}{$\mathbf{p}$} \\
\hline & No & Yes & \\
\hline Age (years) (mean \pm SD) & $63.67 \pm 6.17$ & $64.51 \pm 6.67$ & 0.313 \\
\hline PSA (ng/mL) (median) (min-max) & $7.15(3.06-38.74)$ & $13.65(3.1-107.32)$ & $<0.001$ \\
\hline \multicolumn{4}{|l|}{ PSA distributions, n (\%) } \\
\hline$<10$ & $164(72.57)^{a}$ & $26(34.21)^{b}$ & \multirow[t]{3}{*}{$<0.001$} \\
\hline $10-20$ & $47(20.8)^{a}$ & $26(34.21)^{b}$ & \\
\hline$>20$ & $15(6.64)^{\mathrm{a}}$ & $24(31.58)^{b}$ & \\
\hline \multicolumn{4}{|l|}{ Digital rectal examination, $\mathrm{n}(\%)$} \\
\hline Positive & $77(34.07)$ & $61(80.26)$ & \multirow[t]{2}{*}{$<0.001$} \\
\hline Negative & $149(65.93)$ & $15(19.74)$ & \\
\hline \multicolumn{4}{|l|}{ Biopsy GS, n (\%) } \\
\hline$\leq 6$ & $178(78.76)^{\mathrm{a}}$ & $29(38.16)^{b}$ & \multirow[t]{3}{*}{$<0.001$} \\
\hline 7 & $42(18.58)^{a}$ & $34(44.74)^{b}$ & \\
\hline$\geq 8$ & $6(2.65)^{\mathrm{a}}$ & $13(17.11)^{b}$ & \\
\hline \multicolumn{4}{|l|}{ D'Amico risk classification, $\mathrm{n}(\%)$} \\
\hline Low & $138(61.06)^{a}$ & $12(15.79)^{b}$ & \multirow[t]{3}{*}{$<0.001$} \\
\hline Intermediate & $68(30.09)^{a}$ & $31(40.79)^{a}$ & \\
\hline High & $20(8.85)^{a}$ & $33(43.42)^{b}$ & \\
\hline \multicolumn{4}{|l|}{ Clinical stage, n (\%) } \\
\hline T1b & $1(0.44)^{\mathrm{a}}$ & $0(0)^{\mathrm{a}}$ & \multirow[t]{6}{*}{$<0.001$} \\
\hline T1c & $148(65.49)^{a}$ & $16(21.05)^{b}$ & \\
\hline T2a & $54(23.89)^{a}$ & $21(27.63)^{a}$ & \\
\hline $\mathrm{T} 2 \mathrm{~b}$ & $11(4.87)^{\mathrm{a}}$ & $14(18.42)^{b}$ & \\
\hline $\mathrm{T} 2 \mathrm{C}$ & $12(5.31)^{\mathrm{a}}$ & $17(22.37)^{b}$ & \\
\hline Т3а & $0(0)^{a}$ & $8(10.53)^{b}$ & \\
\hline ALT (IU/L) (median) (min-max) & $18.5(7-48)$ & $18(6-39)$ & 0.589 \\
\hline AST (IU/L) (median) (min-max) & $20(12-44)$ & $21(14-38)$ & 0.097 \\
\hline De Ritis Ratio & $1(0.6-2.44)$ & $1.21(0.65-3)$ & 0.006 \\
\hline Prostate Weight (g) (median) (min-max) & $50(20-180)$ & $54(22-112)$ & 0.827 \\
\hline \multicolumn{4}{|l|}{ Postoperative GS, n (\%) } \\
\hline$\leq 6$ & $115(50.88)^{a}$ & $10(13.16)^{b}$ & \multirow[t]{3}{*}{$<0.001$} \\
\hline 7 & $96(42.48)^{a}$ & $43(56.58)^{b}$ & \\
\hline$\geq 8$ & $15(6.64)^{\mathrm{a}}$ & $23(30.26)^{b}$ & \\
\hline \multicolumn{4}{|l|}{ Pathological stage, n (\%) } \\
\hline $\mathrm{T} 2 \mathrm{a}$ & $53(23.45)^{a}$ & $2(2.63)^{b}$ & \multirow[t]{5}{*}{$<0.001$} \\
\hline $\mathrm{T} 2 \mathrm{~b}$ & $10(4.42)^{\mathrm{a}}$ & $2(2.63)^{a}$ & \\
\hline $\mathrm{T} 2 \mathrm{c}$ & $111(49.12)^{\mathrm{a}}$ & $16(21.05)^{b}$ & \\
\hline T3a & $36(15.93)^{a}$ & $20(26.32)^{b}$ & \\
\hline T3b & $16(7.08)^{a}$ & $36(47.37) b$ & \\
\hline
\end{tabular}




\begin{tabular}{|c|c|c|c|}
\hline \multirow[t]{2}{*}{ Variables } & \multicolumn{2}{|l|}{ BCR } & \multirow[b]{2}{*}{$\mathbf{p}$} \\
\hline & No & Yes & \\
\hline Tumor volume percent, n (\%) & $8(1-80)$ & $29(1-95)$ & $<0.001$ \\
\hline \multicolumn{4}{|l|}{ Surgical margin status, $\mathrm{n}(\%)$} \\
\hline Positive & $47(20.8)$ & $46(60.53)$ & \multirow[t]{2}{*}{$<0.001$} \\
\hline Negative & $179(79.2)$ & $30(39.47)$ & \\
\hline \multicolumn{4}{|l|}{ Perineural invasion, n (\%) } \\
\hline Absent & $61(26.99)$ & $3(3.95)$ & \multirow[t]{2}{*}{$<0.001$} \\
\hline Present & $165(73.01)$ & $73(96.05)$ & \\
\hline \multicolumn{4}{|l|}{ Lymphovascular invason, $\mathrm{n}(\%)$} \\
\hline Absent & $202(89.38)$ & $41(53.95)$ & \multirow[t]{2}{*}{$<0.001$} \\
\hline Present & $24(10.62)$ & $35(46.05)$ & \\
\hline \multicolumn{4}{|l|}{ Extraprostatic extension, n (\%) } \\
\hline Absent & $183(80.97)$ & $23(30.26)$ & \multirow[t]{2}{*}{$<0.001$} \\
\hline Present & $43(19.03)$ & $53(69.74)$ & \\
\hline \multicolumn{4}{|l|}{ Seminal vesicle invasion, $\mathrm{n}(\%)$} \\
\hline Absent & $211(93.36)$ & $40(52.63)$ & \multirow[t]{2}{*}{$<0.001$} \\
\hline Present & $15(6.64)$ & $36(47.37)$ & \\
\hline \multicolumn{4}{|l|}{ Lymph node involment, n (\%) } \\
\hline Negative & $224(99.12)$ & $63(82.89)$ & \multirow[t]{2}{*}{$<0.001$} \\
\hline Positive & $2(0.88)$ & $13(17.11)$ & \\
\hline \multicolumn{4}{|l|}{ Multifocal PSM, n (\%) } \\
\hline No & $218(96.46)$ & $54(71.05)$ & \multirow[t]{2}{*}{$<0.001$} \\
\hline Yes & $8(3.54)$ & $22(28.95)$ & \\
\hline \multicolumn{4}{|c|}{ Equilavence between biopsy GS and prostatectomy GS, n (\%) } \\
\hline Upgrading & $75(33.2)^{a}$ & $47(61.8)^{b}$ & \multirow[t]{3}{*}{$<0.001$} \\
\hline Downgrading & $10(4.4)^{a}$ & $3(3.9)^{a}$ & \\
\hline Same GS & $141(62.4)^{a}$ & $26(34.2)^{b}$ & \\
\hline
\end{tabular}

with higher De Ritis ratio. We also found that intermediate and high-risk D'Amico classification, LVI, EPE, SVI, PSM and upgrading in pathological specimen independently predicted BCR in multivariable regression analyses. Wang et al. (19) first reported that higher AST/ALT ratio was associated with worse pathological outcomes and higher $B C R$ rates, in line with our results. Many studies have been performed to evaluate the pathological factor predicting recurrence in patients organ-confined $\mathrm{PCa}$ and different results have been obtained. A recent metaanalysis reported that SVI, PSM, EPE, LVI, LNI and PNI had a significant relationship with poor BCR-free survival
(20). These parameters have an important place in patient follow-up. Serum AST and ALT levels are effective markers and widely used to monitor liver functions in clinical practice. ALT is found in the hepatocyte cytoplasm while AST is found in both the hepatocyte cytoplasm and mitochondria. AST is expressed in several organs, whereas ALT is found predominantly only in the liver. Increased metabolism in tumor cells, tissue damage and high tumor cell turnover tend to increase AST rather than ALT (21). Therefore, the De Ritis ratio, which is easily accessible and inexpensive, become a potential biomarker. Some previous studies have demonstrated that De Ritis ratio 

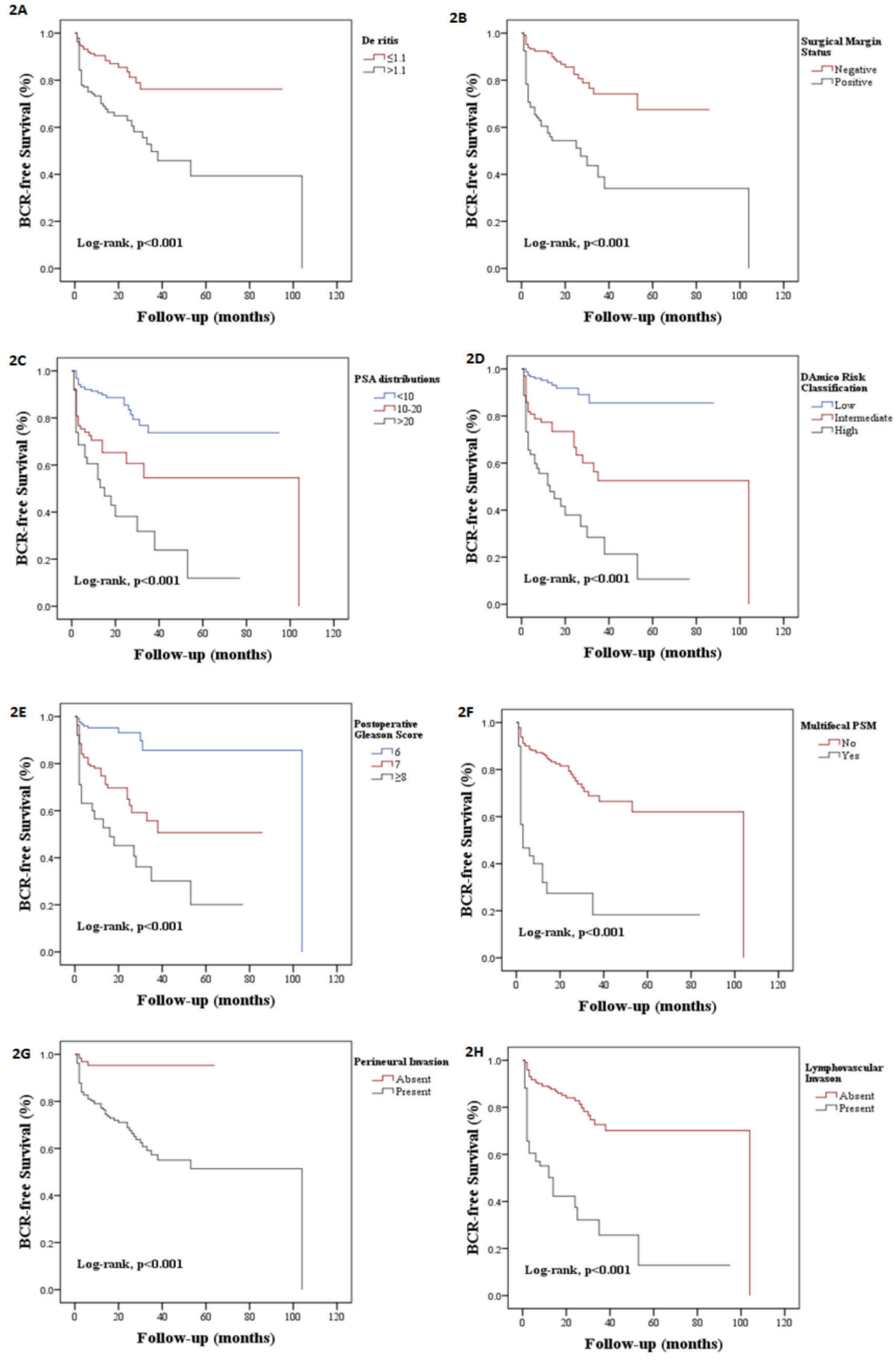

Figure 2. BCR-free survival curves according to eight predictive factors for $B C R$ $B C R$ : Biochemical recurrence 


\begin{tabular}{|c|c|c|c|c|}
\hline \multirow[t]{2}{*}{ Variable } & \multicolumn{2}{|l|}{ Univariate } & \multicolumn{2}{|l|}{ Multivariate } \\
\hline & $\mathrm{HR}(95 \% \mathrm{Cl})$ & p & $\mathrm{HR}(95 \% \mathrm{Cl})$ & $\mathrm{p}$ \\
\hline Age & $1.018(0.981-1.057)$ & 0.346 & - & - \\
\hline PSA & $1.034(1.025-1.044)$ & $<0.001$ & - & - \\
\hline \multicolumn{5}{|l|}{ D'Amico } \\
\hline Low (ref) & - & - & - & - \\
\hline Intermediate & $4.391(2.246-8.586)$ & $<0.001$ & $2.228(1.050-4.728)$ & 0.037 \\
\hline High & $10.679(5.507-20.706)$ & $<0.001$ & $3.316(1.470-7.483)$ & 0.004 \\
\hline DRE (ref: negative) & $5.752(3.216-10.288)$ & $<0.001$ & - & - \\
\hline De Ritis Ratio (ref: $\leq 1.1$ ) & $2.853(1.746-4.664)$ & $<0.001$ & $1.968(1.150-3.368)$ & 0.014 \\
\hline \multicolumn{5}{|l|}{ Clinical stage } \\
\hline T1 (ref) & - & - & - & - \\
\hline T2 & $4.743(2.669-8.429)$ & $<0.001$ & - & - \\
\hline T3 & $27.877(11.63-66.818)$ & $<0.001$ & - & - \\
\hline \multicolumn{5}{|l|}{ Postoperative GS } \\
\hline$\leq 6$ (ref) & - & - & - & - \\
\hline 7 & $5.018(2.444-10.303)$ & $<0.001$ & $1.025(0.375-2.802)$ & 0.961 \\
\hline$\geq 8$ & $9.972(4.609-21.574)$ & $<0.001$ & $0.879(0.276-2.798)$ & 0.827 \\
\hline \multicolumn{5}{|l|}{ Pathological stage } \\
\hline T2 (ref) & - & - & - & - \\
\hline T3 & $6.637(3.94-11.182)$ & $<0.001$ & $0.754(0.185-3.067)$ & 0.693 \\
\hline PNI (ref: absent) & $6.38(2.009-20.266)$ & 0.002 & $1.461(0.403-5.300)$ & 0.564 \\
\hline LVI (ref: absent) & $5.052(3.201-7.975)$ & $<0.001$ & $1.734(1.013-2.966)$ & 0.045 \\
\hline EPE (ref: absent) & $6.601(4.01-10.869)$ & $<0.001$ & $1.970(1.082-3.587)$ & 0.027 \\
\hline SVI (ref: absent) & $6.466(4.09-10.223)$ & $<0.001$ & $1.738(1.007-3.021)$ & 0.048 \\
\hline LNI (ref: absent) & $7.357(4.006-13.511)$ & $<0.001$ & $1.705(0.842-3.453)$ & 0.138 \\
\hline Multifocal PSM (ref: no) & $5.332(3.23-8.804)$ & $<0.001$ & 0.991 (0.497-1.976) & 0.979 \\
\hline \multicolumn{5}{|l|}{ Concordance of GS } \\
\hline Upgrading & $2.662(1.638-4.327)$ & $<0.001$ & $1.670(1.013-2.754)$ & 0.045 \\
\hline Downgrading & $1.984(0.597-6.587)$ & 0.263 & $1.074(0.311-3.707)$ & 0.910 \\
\hline \multicolumn{5}{|l|}{ Same (ref) } \\
\hline Surgical margin status & $4.046(2.547-6.428)$ & $<0.001$ & $1.986(1.201-3.285)$ & 0.008 \\
\hline
\end{tabular}

was a significant prognostic factor in several malignancies (22-25). The mechanism of how the De Ritis ratio predicts poor oncological results remain unclear. Previous studies have pointed out the Warburg effect. Warburg (26) demonstrated that cancer cells tend to show a greater rate of aerobic glycolysis than normal tissue. Fantin et al. (27) observed that lactate dehydrogenase and high $\mathrm{NADH} / \mathrm{NAD}^{+}$ratio were proposed to have an important role in maintaining glucose catabolism. AST has a vital role in glycolysis through the malate-aspartate shuttle pathway that allows NADH/NAD ${ }^{+}$conversion (28). Thus, elevated De Ritis ratio could be indirectly reflecting the glycolysis metabolism. Additionally, Shao et al. (29) investigated the metabolomics and transcriptomics profile in PCa. They observed significant accumulation of metabolic intermediates in tricarboxylic acid (TCA) cycle in tumor tissues, indicating TCA cycle hyperactivation. Accumulation of these metabolites could damage the mitochondria. The efficient energy production in transforming peripheral zone prostate epithelial cells implies increased oxidative 
phosphorylation, and possibly increased mitochondrial reactive oxygen species production (30). We think that elevated De Ritis could be explained by increased aerobic glycolysis, mitochondrial dysfunction and oxidative stress in cancer cells.

We also investigated the association between additional pathological findings and risk of $B C R$ following RP. In a systematic review, PSM has been consistently associated with an increased risk of PSA relapse (31). In our cohort, PSM was an independent predictor factor for BCR. Wu et al. (32) also reported that multifocal PSM had a significant impact on increased $B C R$ risk on univariate analysis and predicting BCR-free survival, in line with our study. A large review has shown that the difference between biopsy GS and prostatectomy GS ranged from $28 \%$ to $58 \%$ (33). In our study, this rate was $40.4 \%$ and upgrading was associated with higher risk of BCR. Upgraded patients should be carefully followed due to increased risk of BCR.

\section{Study Limitations}

Our study had some limitations. First of all, it was a retrospective study with relatively few patients. Secondly, the follow-up period might not have been long enough. Lastly, the De Ritis ratio might have been changed with the presence of undetected liver disease or other diseases. Even with further evidence from future investigations, the optimal De Ritis ratio cutoff value for predicting $B C R$ may be proved. It must be validated in large, prospective studies.

\section{Conclusion}

Our study suggests that elevated De Ritis ratio could be an independent predictive factor for $B C R$ after RP. Additionally, LVI, EPE, SVI, PSM and upgrading can predict the risk of $B C R$. The De Ritis ratio can be easily calculated in routine blood tests and is a cost-effective parameter that can be used in clinical practice to predict BCR following RP. In the postoperative period, the association between the De Ritis ratio and BCR may be the subject of another study.

\section{Authorship Contributions}

Concept: K.K. Design: K.K., A.Y., I.E., E.I. Data Collection or Processing: Y.A., I.E., A.Y. Analysis or Interpretation: Y.A., K.Y., M.A., T.Ö. Literature Search: K.K., K.Y., T.Ö., M.A., M.S. Writing: K.K., E.I., M.S.

Conflict of Interest: No conflict of interest was declared by the authors.

Financial Disclosure: The authors declared that this study received no financial support.

\section{References}

1. Siegel RL, Miller KD, Jemal A. Cancer statistics. CA Cancer J Clin 2015;65:5-29.
2. Hamdy FC, Donovan JL, Lane JA, et al. 10-Year Outcomes after Monitoring, Surgery, or Radiotherapy for Localized Prostate Cancer. N Engl J Med 2016;375:1415-24.

3. Han M, Partin AW, Pound CR, Epstein Jl, Walsh PC. Longterm biochemical disease-free and cancer-specific survival following anatomic radical retropubic prostatectomy: 15-year John Hopkins experince. Urol Clin North Am 2001;28:555-65.

4. Ün S, Türk H, Koca O, Divrik RT, Zorlu F. Factors determining biochemical recurrence in low-risk prostate cancer patients who underwent radical prostatectomy. Turk J Urol 2015;41:616.

5. Han M, Partin AW, Zahurak M, Piantadosi S, Epstein Jl, Walsh PC. Biochemical recurrence probability following radical prostatectomy for clinically localized prostate cancer. J Urol 2003;169:517-23.

6. Diblasio CJ, Kattan MW. Use of nomograms to predict the risk of disease recurrence after definitive local therapy for prostate cancer. Urology 2003;62:9-18.

7. Kotb AF, Elabbady AA. Prognostic factors for the development of biochemical recurrence after radical prostatectomy. Prostate Cancer 2011;2011:485189.

8. Zhang GM, Zhu Y, Ma XC, et al. Pretreatment neutrophil-tolymphocyte ratio: A predictor of advanced prostate cancer and biochemical recurrence in patients receiving radical prostatectomy. Medicine (Baltimore) 2015;94:e1473.

9. Gu X, Gao X, Li X, et al. Prognostic significance of neutrophilto-lymphocyte ratio in prostate cancer:evidence from 16,266 patients. Sci Rep 2016;6:22089.

10. Kang M, Oh JJ, Lee S, Hong SK, Lee SE, Byun SS. Perineural Invasion and Lymphovascular Invasion are Associated with Increased Risk of Biochemical Recurrence in Patients Undergoing Radical Prostatectomy. Ann Surg Oncol 2016;23:2699-706

11. De Ritis F, Coltorti M, Giusti G. An enzymic test for the diagnosis of viral hepatitis: the transaminase serum activities. Clin Chim Acta 1957;2:70-4.

12. Rawson NS, Peto J. An overview of prognostic factors in small cell lung cancer. A report from the Subcommittee for the Management of Lung Cancer of the United Kingdom Coordinating Committee on Cancer Research. Br J Cancer 1990;61:597-604.

13. Lee $H$, Choi $Y H$, Sung $H H$, et al. De Ritis Ratio (AST/ALT) as a Significant Prognostic Factor in Patients With Upper Tract Urothelial Cancer Treated With Surgery. Clin Genitourin Cancer 2017; 15:379-85.

14. Bezan A, Mrsic E, Krieger D, et al. The Preoperative AST/ ALT (De Ritis) Ratio Represents a Poor Prognostic Factor in a Cohort of Patients with Nonmetastatic Renal Cell Carcinoma. J Urol 2015;194:30-5.

15. Thornburg JM, Nelson KK, Clem BF, et al. Targeting aspartate aminotransferase in breast cancer. Breast Cancer Res 2008;10:84. 
16. Walsh PC. Anatomic radical prostatectomy: evaluation of the surgical technique. J Urol 1998;160:2418-24.

17. Briganti A, Larcher A, Abdollah F, et al. Updated nomogram predicting lymph node invasion in patients with prostate cancer undergoing extended pelvic lymph node dissection: the essential importance of percentage of positive cores. Eur Urol 2012;61:480-87.

18. D'Amico AV, Whittington R, Malkowicz SB, et al. Biochemical outcome after radical prostatectomy, external beam radiation therapy, or interstitial radiation therapy for clinically localized prostate cancer. JAMA 1998;208:969-74.

19. Wang $H$, Fang K, Zhang J, et al. The significance of De Ritis (aspartate transaminase/alanine transaminase) ratio in predicting pathological outcomes and prognosis in localized prostate cancer patients. Int Urol Nephrol 2017;49:1391-8.

20. Liu $H$, Zhou $H$, Yan $L$, et al. Prognostic significance of six clinicopathological features for biochemical recurrence after radical prostatectomy: a systematic review and meta-analysis. Oncotarget 2017; 9:32238-49.

21. Conde VR, Oliveira PF, Nunes $A R$, et al. The progression from a lower to a higher invasive stage of bladder cancer is associated with severe alterations in glucose and pyruvate metabolism. Exp Cell Res 2015;335:91-98.

22. Lindmark G, Gerdin B, Pahlman L, et al. Prognostic predictors in colorectal cancer. Dis Colon Rectum 1994;37:1219-27.

23. Stocken DD, Hassan AB, Altman DG. Modelling prognostic factors in advanced pancreatic cancer. $\mathrm{Br} J$ Cancer 2008;99:883-93.

24. Tan X, Xiao K, Liu W, et al. Prognostic factors of distal cholangiocarcinoma after curative surgery: a series of 84 cases. Hepatogastroenterology 2013;60:1892-5.
25. Takenaka Y, Takemoto N, Yasui T, et al. Transaminase Activity Predicts Survival in Patients with Head and Neck Cancer. PLoS One 2016;11:164057.

26. Warburg 0 . On the origin of cancer cells. Science 1956;123:309-14.

27. Fantin VR, St-Pierre J, Leder P. Attenuation of LDH-A expression uncovers a link between glycolysis, mitochondrial physiology, and tumor maintenance. Cancer Cell 2006;9:425-34.

28. Greenhouse WV, Lehninger AL. Occurrence of the malateaspartate shuttle in various tumor types. Cancer Res 1976;36:1392-6.

29. Shao Y, Ye G, Ren S, et al. Metabolomics and transcriptomics profiles reveal the dysregulation of the tricarboxylic acid cycle and related mechanisms in prostate cancer. Int J Cancer 2018;143:396-407.

30. Dakubo GD, Parr RL, Costello LC, Franklin RB, Thayer RE. Altered metabolism and mitochondrial genome in prostate cancer. J Clin Pathol 2006;59:10-6.

31. Yossepowitch O, Briganti A, Eastham JA, et al. Positive surgical margins after radical prostatectomy: a systematic review and contemporary update. Eur Urol 2014;65:303-13.

32. Wu S, Lin SX, Wirth GJ, et al. Impact of Multifocality and Multilocation of Positive Surgical Margin After Radical Prostatectomy on Predicting Oncological Outcome. Clin Genitourin Cancer 2019;17:44-52.

33. King CR, Long JP. Prostate biopsy grading errors: a sampling problem? Int J Cancer 2000;90:326-30. 